9. I. Roman, "An Euler summation formula," Am. Math. Monthly, v. 43, 1936, p. 9-21.

10. H. E. Salzer, "A simple method for summing certain slowly convergent series," $J$. Math and Phys., v. 33, 1954, p. 356-359.

11. H. E. SAlzer, "Formulas for the partial summation of series," $M T A C, \mathrm{v} . \mathrm{X}, 1956$, p. $149-156$.

12. T. B. Sprague, “On Lubbock's formula for approximating to the value of a life annuity," J. Inst. Actuaries, London, v. 18, 1874, p. 305-317.

13. J. F. Steffensen, Interpolation, Williams \& Wilkins, Baltimore, Maryland, 1927, p. $138-148$.

14. E. T. Whittaker, \& G. Robinson, The Calculus of Observations, Blackie, London, 1924, p. 149-150.

\title{
On a Conjecture Concerning the Primes
}

\section{By R. B. Killgrove and K. E. Ralston}

Consider the sequence $\left\{P_{0 j}\right\}, j=0,1,2, \cdots$, where $P_{0 j}$ is the $j$ th prime number, $P_{00}=2, P_{01}=3, P_{02}=5, \cdots$. Now define the absolute differences of the primes by the recursion relation

$$
P_{i j}=\left|P_{i-1, j+1}-P_{i-1, j}\right| \text {. }
$$

The conjecture (Norman L. Gilbreath, private communication, July 1958) is then that $P_{i 0}=1$ for all $i>0$. The validity of the conjecture for the first few primes can be seen from the following table of their absolute differences.

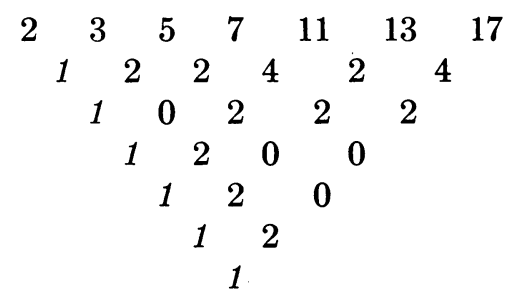

There are an uncountable number of sequences $\left\{b_{0 j}\right\}$ with the property that their absolute differences $b_{i 0}$ defined as above are unity. In particular the sequences $\{k+1, k, k, \cdots\}$ and any sequence of the form $\left\{b_{00}=1 ; b_{0 j}=0\right.$ or $\left.2, j>0\right\}$ have this property. Furthermore it can easily be verified that any sequence, $\left\{b_{0 j}\right\}$, with the required property has its first absolute differences bounded by the sequence $\left\{2^{j}\right\}$, that is, $b_{1 j} \leqq 2^{j}$.

Consider again the absolute differences of the primes. Since all primes greater than 2 are odd numbers it follows that all differences $P_{i j}, j>0$, are even numbers. Now, if for some $i$ and all $j, 0<j<M$, we have $P_{i j}=0$ or 2 and $P_{i 0}=1$, then all of the differences that derive from them will be bounded by 2 , from which it follows that

$$
P_{i, 0}, P_{i+1,0}, P_{i+2,0}, \cdots, P_{i+M-1,0}=1 .
$$

We now define the function $P(i)$ to be the largest integer $M$ such that $P_{i j} \leqq 2$ for all $j<M$. Thus we can say that $P_{k 0}=1$ for $i \leqq k<P(i)+i$.

A routine was coded for the SWAC to evaluate this function $P(i)$, using the primes less than 792,722 from a sieve prepared by $D$. H. Lehmer. The results of

Received Oct. 7, 1958. The preparation of this paper was sponsored by the Office of Naval Research. Reproduction in whole or in part is permitted for any purpose of the United States Government. 
this calculation are shown in the following table. From these results it is seen that the conjecture holds for all primes less than 792,722, which amounts to the first 63,419 primes.

Table of the Function $P(i)$ for $0<i<95$

\begin{tabular}{|c|c|c|c|c|c|c|c|c|}
\hline$i$ & $P(i)$ & $P(i)+i$ & $i$ & $P(i)$ & $P(i)+i$ & $i$ & $P(i)$ & $P(i)+i$ \\
\hline 1 & 3 & 4 & 33 & 867 & 900 & 65 & 23266 & 23331 \\
\hline 2 & 8 & 10 & 34 & 866 & 900 & 66 & 23265 & 23331 \\
\hline 3 & 14 & 17 & 35 & 2180 & 2215 & 67 & 23264 & 23331 \\
\hline 4 & 14 & 18 & 36 & 2179 & 2215 & 68 & 23263 & 23331 \\
\hline 5 & 25 & 30 & 37 & 2178 & 2215 & 69 & 31500 & 31569 \\
\hline 6 & 24 & 30 & 38 & 2177 & 2215 & 70 & 31499 & 31569 \\
\hline 7 & 23 & 30 & 39 & 2771 & 2810 & 71 & 31498 & 31569 \\
\hline 8 & 22 & 30 & 40 & 2770 & 2810 & 72 & 31497 & 31569 \\
\hline 9 & 25 & 34 & 41 & 2769 & 2810 & 73 & 31528 & 31601 \\
\hline 10 & 59 & 69 & 42 & 2768 & 2810 & 74 & 31527 & 31601 \\
\hline 11 & 98 & 109 & 43 & 2767 & 2810 & 75 & 31526 & 31601 \\
\hline 12 & 97 & 109 & 44 & 2766 & 2810 & 76 & 31526 & 31602 \\
\hline 13 & 98 & 111 & 45 & 2765 & 2810 & 77 & 31528 & 31605 \\
\hline 14 & 97 & 111 & 46 & 2764 & 2810 & 78 & 31527 & 31605 \\
\hline 15 & 174 & 189 & 47 & 2763 & 2810 & 79 & 31526 & 31605 \\
\hline 16 & 176 & 192 & 48 & 2763 & 2811 & 80 & 31526 & 31606 \\
\hline 17 & 176 & 193 & 49 & 2763 & 2812 & 81 & 31536 & 31617 \\
\hline 18 & 176 & 194 & 50 & 2763 & 2813 & 82 & 31535 & 31617 \\
\hline 19 & 176 & 195 & 51 & 3366 & 3417 & 83 & 31534 & 31617 \\
\hline 20 & 291 & 311 & 52 & 4208 & 4260 & 84 & 31533 & 31617 \\
\hline 21 & 290 & 311 & 53 & 4207 & 4260 & 85 & 31532 & 31617 \\
\hline 22 & 289 & 311 & 54 & 4206 & 4260 & 86 & 31531 & 31617 \\
\hline 23 & 740 & 763 & 55 & 4205 & 4260 & 87 & 31538 & 31625 \\
\hline 24 & 874 & 898 & 56 & 4204 & 4260 & 88 & 31537 & 31625 \\
\hline 25 & 873 & 898 & 57 & 5943 & 6000 & 89 & 31536 & 31625 \\
\hline 26 & 872 & 898 & 58 & 5944 & 6002 & 90 & 31535 & 31625 \\
\hline 27 & 873 & 900 & 59 & 5943 & 6002 & 91 & 31534 & 31625 \\
\hline 28 & 872 & 900 & 60 & 5942 & 6002 & 92 & 31535 & 31627 \\
\hline 29 & 871 & 900 & 61 & 5941 & 6002 & 93 & 31534 & 31627 \\
\hline 30 & 870 & 900 & 62 & 5940 & 6002 & 94 & 31533 & 31627 \\
\hline 31 & 869 & 900 & 63 & 5940 & 6003 & 95 & $\geq 63324$ & $\geq 63419$ \\
\hline 32 & 868 & 900 & 64 & 5940 & 6004 & 96 & - & - \\
\hline
\end{tabular}

University of California, Los Angeles

\title{
Calculating the Coefficients of Certain Linear Predictors
}

\author{
By D. S. Stoller and L. C. Stoller
}

It is assumed that observations, $x_{j}$, have been made at the $n+1$ points, $j=$ $0,1, \cdots, n$, which are equally spaced. It is desired to find a linear predictor

$$
y_{n+1}=a_{0} x_{0}+\cdots+a_{n} x_{n}
$$

Received 28 September 1958. 